\title{
Feasibility analysis on radiant transient heat flux meter calibration system
}

\author{
ChunChen Sheng ${ }^{1}$, Peng $\mathrm{Hu}^{1, \mathrm{a}}$ and XiaoFang Cheng ${ }^{1}$ \\ ${ }^{1}$ Department of Thermal Science and Energy Engineering, University of Science and Technology of China, \\ 230027Hefei, China
}

Keywords: heat flux meter, calibration, uncertainty, temperature distribution, heat flux non-uniformity.

\begin{abstract}
A heat flux meter calibration system composed of a vacuum cryogenic container, a blackbody furnace and a set of T-type thermocouples measurement system is introduced in this paper. Primary purpose of this system is to calibrate specific heat flux meters applied in spacecraft thermal ground tests within which the heat flux range is from $60 \mathrm{w} / \mathrm{m}^{2}$ to $1400 \mathrm{w} / \mathrm{m}^{2}$ and vacuum is necessary. To simulate space condition, the vacuum container is designed according to the space simulator working standard in thermal ground tests. The required temperature measurement uncertainty in the heat flux meter calibration system is less than $0.1 \mathrm{~K}$ and thermocouple calibration is completed in a more precise thermostatic bath of uncertainty less than $3 \mathrm{mK}$. Thermal character of the vacuum container and the blackbody furnace are verified by NX I-Deas simulations. Besides, Heat flux distribution non-uniformity of the blackbody furnace aperture is $3.5 \%$ and obtained by ray trace method. Consequently, heat flux meter calibration uncertainty less than $5 \%$ is proved to be realizable in this system.
\end{abstract}

\section{Introduction}

General radiant heat flux meters used in various research fields can be divided into two categories when reverse numerical method ${ }^{[1],[2]}$ is absent, namely the heat flux meter based on linear response of the input heat flux and the output signal such as the Gardon meter ${ }^{[3]}$ and the SB meter ${ }^{[4]}$, and the heat flux meter based on non-linear principle such as the plate thermometer ${ }^{[5],[6]}$. Stable radiation source and heat flux meter working surrounding condition are two basic essentials in heat flux meter calibration process, which is mentioned in NIST heat flux meter calibration programs ${ }^{[7]}$.

Heat flux meter for spacecraft thermal test can also be divided into the above two categories: linear type $^{[8]}$ and non-linear type ${ }^{[9],[10],[11],[12]}$. Appropriate calibration of radiant heat flux meters are required to acquire accurate measurement of simulated radiant heat flux in aerospace ground tests, in which the above two essentials of stable and reliable radiant source and heat flux meter working condition are also vital. Consequently, calibration of heat flux meters for thermal test should be completed in the same condition as that of ground thermal test where the radiant heat flux meters to be calibrated are applied ${ }^{[13]}$. Vacuum condition simulation, cold-black environment simulation and space outer heat flux simulation ${ }^{[14]}$ are the primary components of space thermal condition applied in thermal ground tests of spacecraft, which are also prerequisite in radiant heat flux meter calibration. Commonly used

${ }^{\text {a } C o r r e s p o n d i n g ~ a u t h o r ~: ~ h u p e n g @ u s t c . e d u . c n ~}$ 
facility to provide vacuum and cold-black environment in thermal test is heat $\operatorname{sink}^{[15]}$ derived from plates covered by black paint and refrigerated by cryogenic liquid and encapsulated in a vacuum container. This paper describes a calibration system consisting of vacuum heat sink, temperature measurement system and a black-body furnace providing infrared heat flux.

\section{Calibration container design}

In actual space condition, cosmic background radiation is equal to radiant energy emitted by blackbody of $3 \mathrm{~K}$ and the absorptivity of the cosmic space is given as close to 1 . Simulating $3 \mathrm{~K}$ thermal background radiation is implemented by the application of liquid helium refrigerated heat sink, which is unacceptable and cost consuming in large numbers of repeated experiments. Typically, alternative solution is to replace liquid helium by liquid nitrogen under condition of satisfying the test accuracy ${ }^{[14]}$. Temperature of liquid nitrogen refrigerated heat sink covered by black paint of absorptivity higher than 0.9 is about $100 \mathrm{~K}$, limiting the thermal simulating error within $1 \%$ to $2 \%$ when the total area of the heat sink is several times larger than the spacecraft to be tested ${ }^{[16]}$. The same principle is also practicable in the design of heat flux meter calibration system. However, independent heat sink separated from vacuum container commonly found in ground test of whole-satellite ${ }^{[17]}$ is inapplicable to the small calibration container due to its additional process cost and the restrict of the vacuum container volume. As a result, the calibration container internal face covered with black paint is taken as an integrated heat sink by immersing the whole container into liquid nitrogen. Besides, materials application of vacuum calibration container needs to be reconsidered for the design should satisfy both the airtight performance and the property of heat sink simultaneously. Copper, aluminum and stainless steel are three commonly used materials in vacuum technique and cryogenic engineering as a result of their excellent cryogenic property derived from the face-centered cubic crystal structure, consequently comparison of their physical properties ${ }^{[18]}$ indicates that the most appropriate choice of material is stainless steel. The structure of the vacuum container is shown in Fig.1. The main frame of the vacuum container is composed of two separate parts which are assembled by a sealing flange and several bolts to keep high hermetic. Thermocouple cable and heater cable are connected to their monitoring instruments outside the vacuum container by way of a cable channel welded to the top the surface of the upper container. Besides, the branch pipe of the cable channel is linked to a vacuum unit as an air exhausting pipe, keeping the vacuum degree of the whole system $10^{-3} \mathrm{pa}$. The blackbody furnace with the radiation protection surrounding it is hanged by a steel beam mounted below the upper container. Conductive and radiative heat losses from the blackbody furnace are reduced to a low level by the steel beam and the radiation protection shield. The calibrated heat flux meter is placed right below the cavity of the blackbody furnace. Adjusting the sensing foil of the heat flux meter at the same horizontal level with the blackbody furnace cavity would contribute to maintain relative view factor at 1 and promote calibration accuracy.

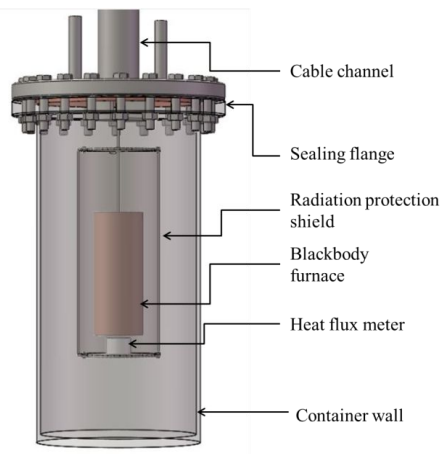

Figure 1. Heat flux meter calibration container 


\section{Analysis on temperature measurement system}

The primary values to be measured in thermal tests are the temperatures of the spacecraft or heat flux meter and the experiment facilities. Even the heat flux measured by a well calibrated heat flux meter is deduced based on the accurately measured temperatures. Therefore, application of proper temperature measurement is another vital aspect to be considered in the heat flux meter calibration system. Advanced temperature measurement systems including wireless measurement and infrared measurement system are gaining increasing number of attentions in spacecraft tests as substitutes of traditional temperature measurements such as thermocouple and $\mathrm{RTD}^{[19]}$. However, based on the requirement of proper temperature measurement range, low cost, reliability and stability of sensor function in vacuum condition, applicability in surface temperature measurement, high sensitivity and small thermal inertia in dynamic measurement and accessibility in installation and swap, thermocouple temperature measurement system is applied in heat flux meter calibration system. Typically used T-type thermocouple in spacecraft thermal test is a priority selection as a result of the identical temperature measurement range of $70 \mathrm{~K}$ to $400 \mathrm{~K}$ in heat flux meter calibration with that in thermal test. Consequently, final choice of temperature measurement scheme mainly consisting of Ttype thermocouples, reference point, and Agilent 34970A data acquisition instrument is determined after the comprehensive consideration of all aspects. Temperature measurement by thermocouple based on thermoelectric effect is supported and restrained by law of intermediate metal and law of intermediate temperature. Except for accurate reference point temperature, measurement cable is another pivotal part in measurement system considering the above two laws, which results in the application of AF-200 type cable with Teflon insulation. Reliable and accurate temperature measurement by thermocouple depends on thermocouple processing technique and precise calibration. Since the quality of the adoptive T-type thermocouple (Omega Corporation) is guaranteed by the supplier, accurate thermocouple calibration is the last essential condition for all accurate measurement in heat flux meter calibration. Output terminal of thermocouple in original measurement system is millivoltmeter which displays the thermoelectric force of the thermocouple in temperature measurement, and the finally acquired temperature value is gained by referencing thermocouple indexing table. Accurate calibration aiming at acquiring proper fitted relationship between temperature value and thermoelectric force before temperature measurement is always necessary in all thermocouple measurement systems.

Agilent 34970A data acquisition instrument of six and a half with an uncertainty of less than $\pm 1 \mathrm{mK}$ is applied as the output terminal of heat flux meter calibration system, which is capable of displaying temperature values directly. However, accuracy and reliability of thermocouple temperature measurement should also be taken seriously for purpose of accurate heat flux meter calibration. Solutions to verify the temperature measurement accuracy is based on comparing the displayed temperature value with the known accurate temperature value. Finally, experiment facility applied in paper ${ }^{[20]}$ is adopted in thermocouple calibration. The experiment apparatus composed of a thermostatic bath, a temperature measurement and control system, a pressure measurement system, a vacuum system and an optical sample cell is primarily designed for refrigerant vapor and liquid equilibrium experiment, which possesses a total temperature-control uncertainty of $\pm 2 \mathrm{mK}$. A fourwire 25- $\Omega$ platinum resistance thermometer (Yunnan Instrument) with an uncertainty of $\pm 2 \mathrm{mK}$ (ITS90 ) is firstly calibrated by the water's triple-point temperature and then applied in the temperature measurement and control system, making the overall temperature uncertainty $\pm 5 \mathrm{mK}$. Incremental digital PID algorithm is applied in the temperature measurement program capable of measuring and controlling the temperature of the thermostatic bath simultaneously in a large temperature range from $233.15 \mathrm{~K}$ to $375.15 \mathrm{~K}$ based on the application of variable bath fluid.

According to thermocouple function principle, reference point temperature is the precondition of accurate calibration and measurement. Commonly used reference point in thermocouple measurement is ice and water mixture with ideal temperature of $0{ }^{\circ} \mathrm{C}$. However, in laboratory environment, the actual temperature of ice and water mixture may differ from the ideal temperature. As a result, the temperature of applied ice and water mixture is monitored by another four-wire $25-\Omega$ platinum 
resistance thermometer. Temperature field distribution of the homogeneously mixed ice and water contained in a well performing thermostatic vessel is verified by changing the RTD measurement position several times. Temperature values of 50 different measurement point positions shown in Fig.2. Type A evaluation of standard uncertainty based on statistical method ${ }^{[21],[22]}$ is applied in determining the reference thermostatic vessel temperature uncertainty which is also known as standard deviation, as shown in Eq.(1)

$$
u\left(x_{i}\right)=s\left(\overline{X_{i}}\right)=\left[\frac{1}{n(n-1)} \sum_{k=1}^{n}\left(X_{i, k}-\frac{1}{n} \sum_{k=1}^{n} X_{i, k}\right)^{2}\right]^{1 / 2}
$$

where $\mathrm{n}$ is the size of sample space. The type A standard uncertainty of the out reference point temperature is less than $1 \mathrm{mK}$. Consequently, thermocouple calibration uncertainty caused by the reference point temperature measured by the four-wire $25-\Omega$ platinum resistance thermometer in the ice and water mixture is less than $0.01 \mathrm{~K}$ considering the temperature field distribution of the mixture and the four-wire $25-\Omega$ platinum resistance thermometer.

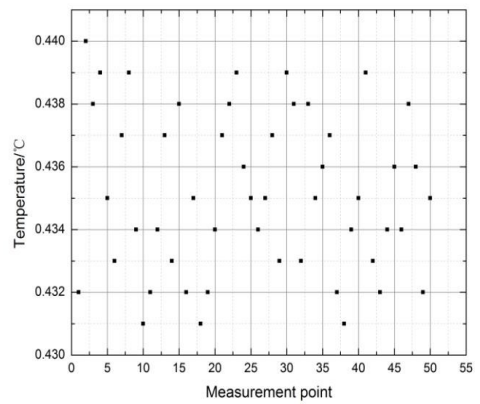

Figure 2. Temperature of ice and water mixture in laboratory environment

Since the reliability of the reference point temperature and the thermostatic bath temperature are ensured, thermocouple calibration in the thermostatic bath shall be conducted without any concerns of actual temperature value. The demanded temperature of the thermostatic bath varies from $278.15 \mathrm{~K}$ to $363.15 \mathrm{~K}$ with increment of $5 \mathrm{~K}$ depending on the regulated heater and evaporator ruled by the PID temperature control program. Under any regulated temperature value when the thermostatic bath achieves steady state, thermocouple calibrated temperature value averaged from 5 different thermocouple measurement positions is shown in Fig.3.

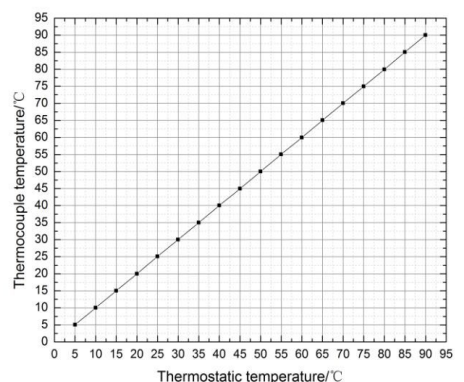

(a)Temperature calibration

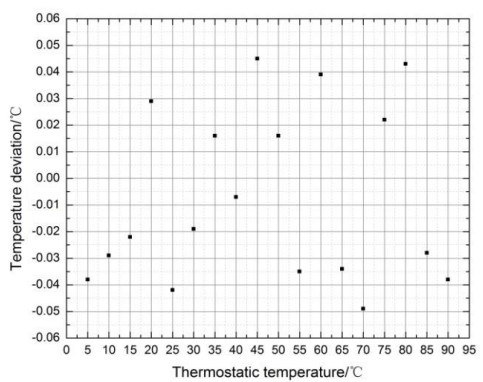

(b)Temperature deviation

Figure 3. Thermocouple calibration by thermostatic bath

As is shown in Fig.3(b), average correction for thermocouple measurement is $7 \mathrm{mK}$ and measurement correction uncertainty is $8 \mathrm{mK}$. Since the standard uncertainty components ${ }^{[22]}$ of the reference point temperature, the thermostatic bath temperature and the thermocouple calibration correction all contribute to the combined uncertainty of thermocouple measurement, the total combined uncertainty of thermocouple measurement is less than $100 \mathrm{mK}$ according to the law of uncertainty propagation ${ }^{[22]}$ displayed in Eq.(2) 


$$
u_{c}^{2}(y)=\sum_{i=1}^{N}\left(\frac{\partial f}{\partial x_{i}}\right) u^{2}\left(x_{i}\right)+2 \sum_{i=1}^{N-1} \sum_{j=i+1}^{N} \frac{\partial f}{\partial x_{i}} \frac{\partial f}{\partial x_{j}} u\left(x_{i}, x_{j}\right)
$$

where the partial derivatives are also referred to as sensitivity coefficients and $\mathrm{u}\left(\mathrm{x}_{\mathrm{i}}, \mathrm{x}_{\mathrm{j}}\right)$ is the estimated covariance associated with $\mathrm{x}_{\mathrm{i}}$ and $\mathrm{x}_{\mathrm{j}}$. Consequently, taking into consideration of other influencing factor such as output terminal uncertainty and the output terminal uncertainty will barely impact on the measurement results since the experiment required uncertainty is two orders of magnitude larger than the thermocouple measurement uncertainty. The excellent linearity of the thermostatic bath temperature and the calibrated thermocouple temperature indicate that the required linearity also exists in other temperature range when the T-type thermocouple would function properly from $-200{ }^{\circ} \mathrm{C}$ to $200^{\circ} \mathrm{C}$.

Ice and water mixture is applied as the thermocouple measurement reference in thermocouple calibration. However, ice point reference is inapplicable to heat flux meter calibration system. Commonly used reference point in vacuum thermal test is an internal separated device containing all thermocouple reference endpoints and measured by a four-wire $25-\Omega$ platinum resistance thermometer, playing a significant role in the reliability and accuracy of the whole thermocouple measurement system. Consequently, stable, reliable and uniform temperature field capable of being accurately measured is required in separated reference point device to aid thermocouple measurement. Separated reference point device in vacuum thermal test is mounted inside the environment simulator, temperature field unevenness of which is less than $0.1 \mathrm{~K}^{[23]}$. However, the introduced reference point device used in vacuum thermal tests is inapplicable in heat flux meter calibration system for the same reason as the inapplicability of independent heat sink. Consequently, heat flux meter calibration container internal surface is regarded as a candidate of the separated reference point device.

\section{Analysis on reference point, heat sink and blackbody furnace}

The feasibility of taking the vacuum container inner surface as the reference point needs to be verified beforehand to ensure the thermocouple measurement accuracy. During the calibration process when the blackbody furnace temperature is stable at $312 \mathrm{~K}$, the temperature distribution graph of the inner bottom surface is shown in Fig.4. Obviously, temperature distribution of the calibration container bottom surface is affected by the cavity of the heating blackbody furnace which is pasted with aluminized polyimide membrane with emissivity of 0.1 on its side face and top face to eliminate additional thermal radiation leak. The temperature unevenness of the whole bottom surface is about $5 \mathrm{~K}$ and the isotherms of this surface are series of concentric circles around the central point. Reference endpoints of the working thermocouples and the platinum resistance thermometer measurement point on the bottom surface have to be mounted precisely along the centric circles around the center to acquire identical reference temperatures, which is unmanageable in actual experiments and may cause unacceptable errors by a tiny position deviation. As a result, mounting the reference endpoints of the thermocouples measuring the blackbody furnace temperature and the heat flux meter sensing foil temperature on the bottom surface is impracticable and unreliable resulting in the outcome of inevitable calibration errors of the heat flux meter.

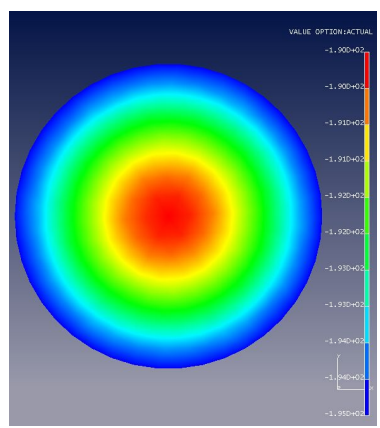

Figure 4. Temperature distribution of vacuum container bottom wall, blackbody furnace at $312 \mathrm{~K}$ 
However even if the temperature distribution of the calibration container side wall surface is similar to that of the bottom surface, implement of the reference point on the side wall is achievable. According to Fig.5(a), isothermal points of the wall surface are distributed at the same height from the bottom plane and the unevenness of the isotherm is less than $0.01 \mathrm{~K}$, conforming to the function feature of the additional reference point device. Besides, even if the maximum or minimum temperature of the side wall would change somehow when the inner radiative heating source is regulated to calibrate the heat flux meter under different heat flux range, the isotherm distribution of the side wall would stay unchanged as a result of the fixed location relationship between the radiative source and the vacuum container, which is certified by the comparison results of different calibration heat flux when the temperatures of inner heating source are $398 \mathrm{~K}$ and $176 \mathrm{~K}$ shown in Fig.5(b) and Fig.5(c). Consequently, mounting the thermocouple reference endpoint at the same height as the fourwire platinum resistance thermometer is a feasible solution instead of the separate reference point device in vacuum thermal tests. In actual operation, total thermocouple temperature measurement uncertainty considering height measurement uncertainty in positioning the reference point is less than $0.1 \mathrm{~K}$. Besides, the minimum temperature of the inner surface of the calibration container is lower than $100 \mathrm{~K}$ and the absorptivity of the inner surface is nearly 1 , basically according with the definition of commonly used heat sink in vacuum thermal tests of spacecraft. The temperature uniformity of the entire vacuum container caused by the heating blackbody furnace in heat flux meter steady state calibration shall be reduced by the additional radiation protection shield surrounding the blackbody furnace.

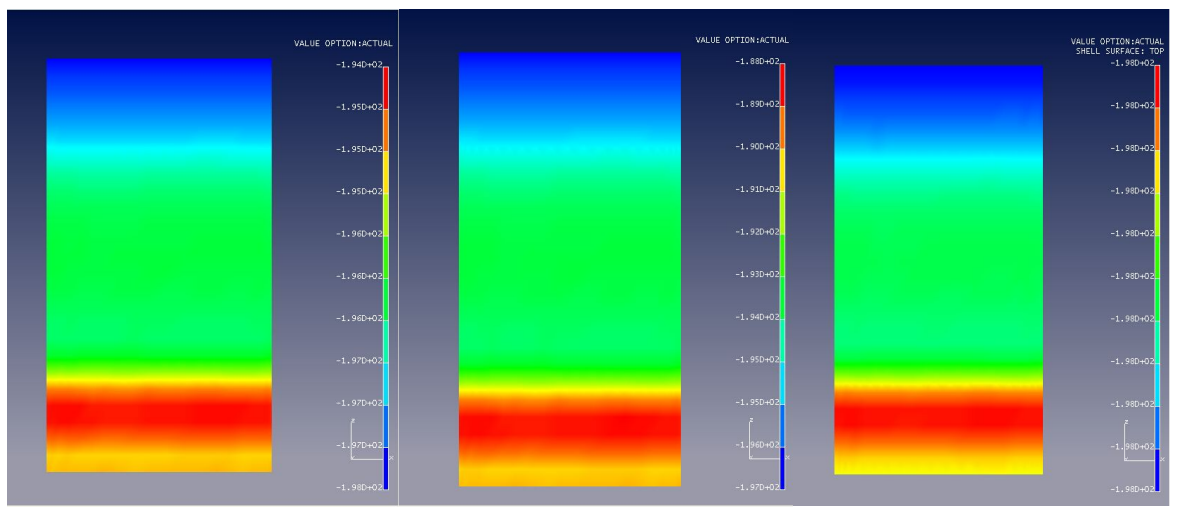

(a) $312 \mathrm{~K}$

(b) $398 \mathrm{~K}$

(c) $176 \mathrm{~K}$

Figure 5. Temperature distribution of container side wall at different blackbody furnace temperature

The basic purpose of heat flux meter calibration for either type is to establish an appropriate mathematic relationship between the income heat flux received by the heat flux meter and the heat flux meter temperature or the output signal, as shown in Eq.(3)

$$
q^{\prime \prime}=f(T, \mathrm{~d} T / \mathrm{d} t)
$$

where $\mathrm{q}$ is the known income radiant heat flux, $\mathrm{T}$ is the heat flux meter sensing foil temperature, time derivative term is zero when steady state is achieved. Naturally, the last significant component of the heat flux meter calibration system is the inner radiative heating source which is capable of providing variable and constant radiative heat flux. Commonly used radiative heating sources in spacecraft thermal tests include infrared lamp array and infrared heating cage. However, the accurate heat flux emitted by such facilities has to be measured by another standard radiant heat flux meter. Too many limitations exists in the application of infrared lamp and infrared heating cage as the radiative heating source in this heat flux meter calibration system on account of their non-uniform radiation distribution. Heat flux meter calibration of transfer method ${ }^{[7]}$ in NIST also requires an accurate standard heat flux meter in the same location and working condition as the calibrated heat flux meter to measure the actual radiant heat flux, which is unsuitable for calibration in sealed vacuum container. However, according to the theory of man-made blackbody, artificial cylindrical blackbody furnace is the best option to provide known uniform radiation in heat flux meter vacuum container calibration. Therefore, 
the applied artificial blackbody furnace is made of copper and sprayed with black paint with emissivity of 0.98 on its inner surface. The effective emissivity of the blackbody furnace cavity is estimated to be 1 and independent of furnace temperature by the deduction of both the blackbody furnace structure analysis and Monte Carlo method ${ }^{[24]}$. Basically, the uniform temperature distribution of blackbody furnace is the prior condition to provide uniform radiation for the calibrated heat flux meter and to calculate the theoretical radiant heat flux by Eq.(4)

$$
q=\varepsilon \sigma T^{4}
$$

where $T$ is the average temperature of the blackbody furnace, $\sigma$ is Stefan-Boltzmann constant and $\varepsilon$ is the effective emissivity of blackbody furnace cavity. In addition, more reliable and accurate mathematic relationship between the furnace temperature and the emitted radiant heat flux can also be obtained by standard heat flux meter calibration method as a substitution of Eq.(4) if necessary. Actual method to change the blackbody furnace temperature is done by regulating the input voltage of the thin-film heating attached on the cylindrical side wall. Uniform temperature distribution of the blackbody furnace is the key condition in providing uniform radiative heat flux and acquiring the exact value of the provided heat flux. During the heat flux meter working range from $60 \mathrm{w} / \mathrm{m}^{2}$ to $1400 \mathrm{w} / \mathrm{m}^{2}$ when the correspondent blackbody furnace temperature is from about $176 \mathrm{~K}$ to $293 \mathrm{~K}$, three representative temperature distribution graphs are shown in Fig.6(a), Fig.6(b) and Fig.6(c). The total unevenness of the temperature distribution in any state is less than $0.01 \mathrm{~K}$. Consequently, the actual radiation heat flux uncertainty in calibration is less than $0.1 \mathrm{w} / \mathrm{m}^{2}$ according to Eq.(4) and Eq.(2).

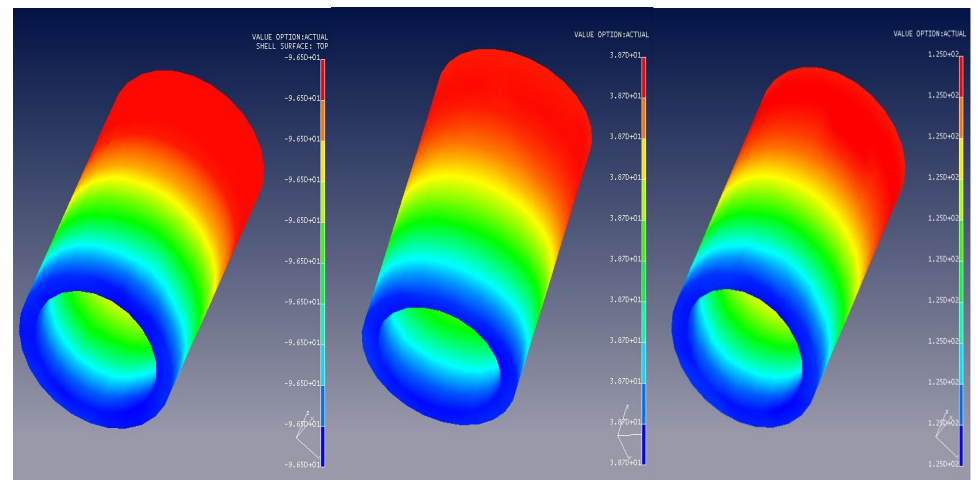

(a) $176 \mathrm{~K}$

(b) $312 \mathrm{~K}$

(c) $397 \mathrm{~K}$

Figure 6. Blackbody furnace temperature distribution at different temperatures

Actual heat flux distribution uniformity of the blackbody furnace aperture is verified by ray tracing method in Tracepro software. The number of the rays is $10^{6}$. The radius of the black body furnace aperture is $45 \mathrm{~mm}$. As is shown in Fig.7, when the furnace wall temperature is $350 \mathrm{~K}$ and perfectly uniform, heat flux distribution of the aperture is acceptable, especially in the vicinity of the center of the aperture.
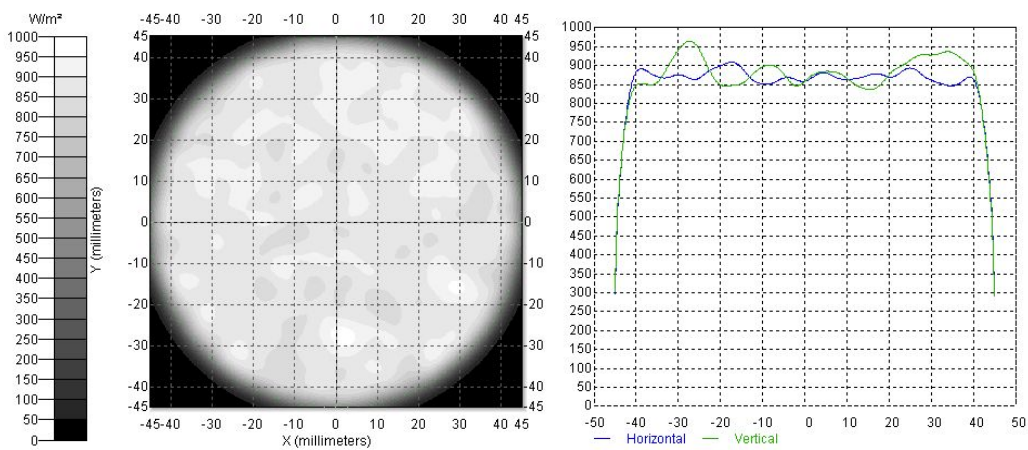

Figure 7. Heat flux distribution of the blackbody furnace aperture when the furnace wall temperature is uniform 
Judging from the vertical and the horizontal heat flux curves, the total heat flux distribution nonuniformity ${ }^{[25]}$ is $3.5 \%$ and appropriate for calibrating heat flux meter with radius of $15 \mathrm{~mm}$ according to Eq.(5):

$$
\text { Non-uniformity }(\%)=\left[\frac{\max \text { irradiance }-\min \text { irradiance }}{\max \text { irridiance }+\min \text { irradiance }}\right] \times 100 \%
$$

where max irradiance and min irradiance are the maximum and minimum heat flux values in the heat flux meter sensing foil area of the absorbing panel.

During actual experiment operation, temperature distribution of the furnace wall is hardly uniform as a result of thermal properties of the construction material, which is displayed in Fig.6. According to the temperature distribution trend of the simulation results, heat flux distribution of the aperture is similar with that of Fig.7 given the maximum temperature difference is $10 \mathrm{~K}$, which is the worst situation and hardly met. The heat flux distribution non-uniformity in the heat flux meter area is $4.8 \%$ and still acceptable compared to the result in Fig.7, giving a large threshold for the furnace wall temperature distribution to provide acceptable heat flux in the furnace aperture.

Due to machining uncertainty and length measurement uncertainty, it is of high probability that the heat flux meter sensing foil and the furnace aperture are noncoplanar. Obviously, uniformity of the heat flux received by the heat flux meter sensing foil needs to be questioned. However, when the absorb panel is $5 \mathrm{~mm}$ away from the aperture, heat flux distribution non-uniformity is $4 \%$ in the heat flux meter sensing foil area and decreases when the absorbing panel gets closer to the furnace aperture. Therefore, simulation result of heat flux distribution indicates that the large threshold of positioning uncertainty keeps such concerns away.

\section{Conclusion}

Since the radiant heat flux meter is used in heat flux measurement of spacecraft thermal tests in space environment simulator, heat flux meter calibration experiments should be completed in the same environment. The feasibility of the heat flux meter calibration system composed of a targetedly designed container and an inner radiative heating source to simulate the space cold and black condition and to provide uniform known radiative heat flux is verified by NX I-Deas simulation. The temperature of the vacuum container inner surface is less than $100 \mathrm{~K}$ and the emissivity of the inner surface is 1 by being painted with black coating, according with the working condition of the space environment simulator in spacecraft thermal tests when the vacuum container is immersed in liquid nitrogen and the vacuum degree is $10^{-3} \mathrm{pa}$ during calibration experiments. Heat flux meter calibration error caused by the experiment facility deviation is estimated to be $1 \%$ to $2 \%$ based on the ground thermal tests experiences. Calibration of T-type thermocouple in thermostatic bath of uncertainty less than $2 \mathrm{mK}$ indicates the thermocouple measurement uncertainty is less than $100 \mathrm{mK}$ when the reference point is ice and water mixture of temperature distribution uniformity less than $1 \mathrm{mK}$. Choosing the isotherms on vacuum container side wall as the reference point of thermocouple measurement in heat flux meter calibration system is also proved to be viable for the temperature distribution unevenness at the same height from the bottom is less than $0.01 \mathrm{~K}$. At last, the feasibility of the blackbody furnace as a standard infrared radiative heating source is reliable for the temperature distribution uniformity is better than $0.01 \mathrm{~K}$ and the calibration radiation heat flux uncertainty is less than $0.1 \mathrm{w} / \mathrm{m}^{2}$. Moreover, heat flux distribution of furnace aperture is clarified by Tracepro software and the non-uniformity of the heat flux meter sensing foil area in the furnace aperture is $3.5 \%$. Thresholds of the temperature wall distribution and the distance between the absorbing panel and the furnace aperture to limit the heat flux distribution non-uniformity of the absorbing panel within 5\% are large and easily-acquired in current laboratory condition. Consequently, the heat flux meter calibration system is proved to be feasible and capable of limiting the total relative calibration uncertainty within $5 \%$ by correct experiment control. 


\section{Acknowledgment}

This work is supported by the National Natural Science Foundation of China (Grant No.:51576187).

\section{References}

1. R. Kumar, N. Sahoo: J. Heat. Trans-t ASME Vol. 135 (2013), p. 123502-1

2. H. Mohammed, H. Salleh: Int. Commun. Heat. Mass Vol. 35 (2008), p. 853

3. R. Gardon : Rev. Sci. Instrum Vol.24 (1953), p. 366

4. R. Bryant, C. Womeldorf and E. Johnsson: Fire. Mater Vol. 27 (2003), p. 209

5. H. Ingason, U. Wickstrom : Fire. Safety. J Vol. 42 (2007), p. 161

6. A. Haggkvist, J. Sjostrom and U. Wickstrom: J. Fire. Sci Vol. 31 (2012), p. 166

7. A.V. Murthy, B.K.Tsai and C.E.Gibson: J. Res. Natl. Inst. Stan Vol. 102 (1997), p. 479

8. W.H. Duan, T. Yu and Y. Yang: Journal of Rocket Propulsion Vol. 40 (2014), p. 87

9. Q.H. Gao, D.F. Qie: Spacecraft Environment Engineering Vol. 24 (2007), p. 310

10. Y. Jia: Chinese Space Science and Technology No. 2 (2001), p. 54

11. Z.Q. Xing, Q.W. Zhao: Spacecraft Environment Engineering Vol. 31 (2014), p. 522

12. Z.S. Chen, B.Y. Yang and P. Hu, Acta. Energiae. Solaris. Sinica Vol. 27 (2006), p. 754

13. Y. L. Ma: Spacecraft Environment Engineering No. 2 (2001), p. 8

14. J.R. Jing, L.X. Zhang: Spacecraft Environment Engineering Vol. 25 (2008), p. 447

15. D.Z. Zou, M. Liu and G.Q. Liu: Chinese Space Science and Technology No. 3(2002), p. 20

16. M. Liu, D.Z. Zou and G.Q. Liu: Missiles and Space Vehicles No. 1 (2001), p. 43

17. B.C Huang: Spacecraft Environment Engineering Vol. 19 (2002), p. 11

18. L. Wang, W.J. Chen, B.X. Jiao and H.J. Sun: Cryogenic No.4 (2003), p. 1

19. G. Guo: Spacecraft Environment Engineering Vol. 26 (2009), p. 33

20. P. Hu, Z.S. Chen and W.L. Chen: J. Chem. Eng. Data Vol.47 ( 2002), p. 20

21. M.O. McLinden, J.D. Splett: J. Res. Natl. Inst. Stan Vol. 113 (2008), p. 39

22. B.N. Taylor, C.E. Kuyatt: Guidelines for evaluating and expressing the uncertainty of NIST measurement results, NIST/TN-1297, NIST: Gaithersburg, MD, 1994.

23. X.H. Sun, X.M. Su and T. Tao: Spacecraft Environment Engineering, Vol. 29 (2012), p. 522

24. A. Ono: J. Opt. Soc. Am. B Vol. 70 (1980), p. 547

25. International Electrotechical Commission, IEC60904-9, Photovoltaic devices-Part9: Solar simulator performance requiremensts, 2007 